\title{
Klarheit bei den Kosten steigert die Leistung der Spitäler
}

Der Verein SpitalBenchmark bietet eine repräsentative Plattform für den fairen Effizienzvergleich von Spitälern in der Schweiz. Der gewichtete Durchschnitt der Fallkosten, nach der Methode des Preisüberwachers standardisiert und mit der Fallschwere (Diagnosis-related Groups DRG) normiert, betrug im Jahr 2006, ohne die Universitätsspitäler, 8690 Franken. Die Streuung ist beträchtlich, auch in Berücksichtigung der regional unterschiedlichen Rahmenbedingungen. Ein regelmässiger und offener Erfahrungsaustausch zwischen den Spitälern soll die Unterschiede - tendenziell kostensenkend - verkleinern oder begründen. Objektiv vergleichbare Zahlen sind die Voraussetzung für Verbesserungen.

\section{Marc Kohler}

CEO Spital Thurgau AG und Präsident Verein SpitalBenchmark
Korrespondenz: Dr. Marc Kohler Spital Thurgau AG Waldeggstrasse 8a CH-8501 Frauenfeld tel. 0527237850 marc.kohler@stgag.ch

\section{Diskussion mit vergleichbaren Zahlen}

Die Effizienz der Spitäler und die von ihnen verursachten Kosten werden heute in der Schweiz breit und öffentlich diskutiert, allerdings kaum je auf der Grundlage von Zahlen, die sich seriös miteinander vergleichen lassen. Dabei wäre dies unabdingbar, spielt doch nach den jüngsten Beschlüssen der Eidgenössischen Räte die leistungsspezifische Finanzierung im neuen KVG eine zentrale Rolle.

Der Verein SpitalBenchmark, ein Zusammenschluss von gegenwärtig 44 Spitälern in der Schweiz, teilweise in Spitalgruppen, wurde im März 2007 mit einer doppelten Zielsetzung gegründet: Einerseits geht es um die interne Vergleichbarkeit von Fallkostendaten und den Aufbau eines auf Kennzahlen basierten Führungsinstruments. Anderseits sind dem Verein objektive Kostenvergleiche im Tarifverfahren und in öffentlichen Diskussionen wichtig. Der Verein gibt den Mitgliedern Analysen und Hinweise auf Abweichungsursachen sowie Empfehlungen zur Realisierung von Verbesserungspotentialen. Unter gewissen Rahmenbedingungen können einzelne Daten als Referenz für tarifliche und politische Diskussionen herangezogen werden.

Die Voraussetzungen für die Erhebung der Kosten, die Berechnung der Effizienz und für die gesicherte Vergleichbarkeit des Zahlenmaterials sind geschaffen. Methodisch im Vordergrund steht der Benchmark von normierten stationären Fallkosten der einzelnen Spitäler und Spital-

\section{La transparence des coûts aug- mente l'efficacité des hôpitaux}

L'Asocciation SpitalBenchmark est une plate-forme représentative pour une juste comparaison de l'efficacité des prestations hospitalières en Suisse. La moyenne pondérée des coûts par cas, standardisée selon la méthode du Surveillant des prix et normée en fonction de la gravité des cas (DRG, Diagnosis-related Groups), se montait, sans compter les hôpitaux universitaires, à 8690 francs pour 2006. Les écarts sont considérables, et ce même si l'on tient compte des différences régionales dans les conditions-cadres. Un échange d'expériences régulier et ouvert entre les hôpitaux doit permettre de réduire ou d'étayer ces écarts par une diminution des coûts. Une comparaison objective des données est à la base de toute amélioration.

gruppen. Die genaue Berechnung erfolgt aus der Summe aller anrechenbaren Kosten, die durch die Anzahl der stationären Fälle dividiert und mit dem sogenannten Case-Mix-Index (CMI), also der durchschnittlichen Schwere der Patientenfälle, gewichtet wird. 
Mehr Transparenz und höhere Akzeptanz Um Transparenz und Akzeptanz zu erhöhen, werden die anrechenbaren Kosten nach der Methode bestimmt, die der Preisüberwacher bei der Tariffestsetzung anwendet. Sie weicht systematisch vom Verfahren innerhalb des Kantons Zürich ab, wo die Kosten im Vergleich zur Preisüberwachermethode grundsätzlich etwas anders angerechnet werden. Codierungsbasis aller Fälle ist die jeweils gültige Version von AP-DRG. In der Praxis ist die objektive Umsetzung dieser Formel nicht ganz einfach. Markant unterschiedliche Voraussetzungen existieren kostenseitig - genaue Definition und Abgrenzung in der Kostenrechnung -, besonders aber in der Bewertung der einzelnen Fallschweregrade via Codierung.

In der Tat erweist sich die Sicherung eines objektiven und fairen Benchmarkprozesses als aufwendig und anspruchsvoll. In die Leistungsfakturierung muss dazu künftig ausnahmslos der Schweregrad (CMI) der stationären Fälle einfliessen. Das geschieht heute erst teilweise, weil die Abrechnung noch vielfach über die Tages- und Abteilungsfallpauschalen erfolgt.

Jedes Tarifsystem besitzt seine Vor- und Nachteile und beinhaltet meist auch systemimmanente verzerrende Faktoren, woraus auch einseitige Vorteile geschöpft werden können. Deshalb sind präzise und adäquate Codierrichtlinien zwingend erforderlich. Sie müssen systematisch angewendet und durch unabhängige Experten überprüft werden. Unsere Schätzung geht heute im CMI von einem Spielraum von etwa $\pm 3 \%$ rein durch die fachgerechte Codierung analoger Fälle aus.

\section{Das Problem der Aufenthaltsdauer}

Zusätzlich bestehen aber massgebliche medizinische Faktoren, die die Varianz der Codierung im Vergleich aller Spitäler der Schweiz erhöhen. Der wichtigste Einfluss ergibt sich aus der Aufenthaltsdauer, die über die im Codiersystem erfasste maximale Normlänge, den sogenannten High Trim Point (HTP), hinausgeht. Solche Fälle werden im aktuellen DRG-System überproportional bewertet. Der Anteil der Kostengewichte durch «Überlieger», d. h. durch Patienten, die länger als medizinisch notwendig im Spital bleiben müssen, ist ein interessanter Qualitätsindikator für den Behandlungspfad und die Codierung. Die Ursachen für eine zu lange Aufenthaltsdauer liegen allerdings oft nur teilweise bei den Spitälern. Häufig ist es unmöglich - auch bei optimaler Vorbereitung und Vernetzung -, die Patienten nach der erfolgreichen Behandlung, dem erreichten Outcomeziel, an nachfolgende Leistungserbringer zu überweisen. In den Pflegeheimen, die einen wichtigen und hochwertigen Teil der Versorgungskette bilden, können im akuten Zeitpunkt die Plätze fehlen. Auch der Mangel an betreuendem Fachpersonal in den Pflegeinstitutionen wird zunehmend zum limitierenden Faktor.

\section{Zwingend notwendige Datenprüfung}

Der Verein SpitalBenchmark hat die Daten des Basisjahres 2006 seiner Mitglieder erfasst und plausibilisiert. Dies betrifft sämtliche anrechenbaren Kosten wie auch die Codierungsqualität. Für Letztere besteht teilweise noch etwas wenig Erfahrung in den einzelnen Spitälern. Eine Überprüfung dazu basiert auf dem zu erwartenden gesamten CMI, der heute aufgrund der statistischen Daten des Vereins SpitalBenchmark und des Angebotsspektrums eines Leistungserbringers relativ gut abgeschätzt werden kann. Die Abweichung der effektiven Codierungen von diesem erwarteten Wert beträgt in den heute vorliegenden Daten meist unter etwa 5\%, was grundsätzlich auf eine gute, professionelle Codierqualität der Mitgliederspitäler schliessen lässt.

Die Erfahrung zeigt, dass die Prüfung der gelieferten Daten zwingend notwendig ist: Kosten und Codierungen müssen zur effektiven Vergleichbarkeit teilweise umgestellt werden. Über sämtliche Versicherungskategorien und Spitäler gerechnet repräsentieren die heute vorhandenen Daten rund 35\% der 2006 in der Schweiz stationär hospitalisierten Patientinnen und Patienten. Geographisch ist die Schweiz mehrheitlich abgedeckt. Noch nicht vertreten sind allerdings Teile der Westschweiz sowie die Kantone Zürich und St. Gallen. Der Branchenverband $\mathrm{H}+$ ist in die Benchmarkinitiative eingebunden und trägt sie als Passivmitglied mit. Für 2007 werden weitere und namhafte Spitäler ebenfalls ihre Daten liefern. Das bedeutet, dass dann rund $50 \%$ der stationären Fälle der Schweiz bis auf die Kantone Genf, Jura und Appenzell-Innerrhoden mit ihren wichtigsten Institutionen erfasst sind (Abb. 1 und 2).

Vorläufig gehören lediglich öffentliche Spitäler, vor allem Universitäts- und Kantonsspitäler, zum Verein SpitalBenchmark. Sie sind mit ihrem breiten Grundversorgungsauftrag und echtem 24-Stunden-Notfalldienst sehr ähnlich positioniert. Die ersten Diskussionen über den Einbezug von Privatkliniken haben begonnen. Heikel ist dort in erster Linie die Erfassung sämtlicher anrechenbarer Kosten, namentlich von ausgelagerten Dienstleistungen und von Belegärzten, die ihre Honorare direkt an Versicherer oder Versicherte fakturieren. Deshalb sind diese Beträge in den Spitalkosten meist nicht enthalten. 


\section{Abbildung 1}

Nach DRG normierte Fallkosten der Spitäler im Verein SpitalBenchmark (Kostenerfassung nach Methode des Preisüberwachers, Daten 2006).

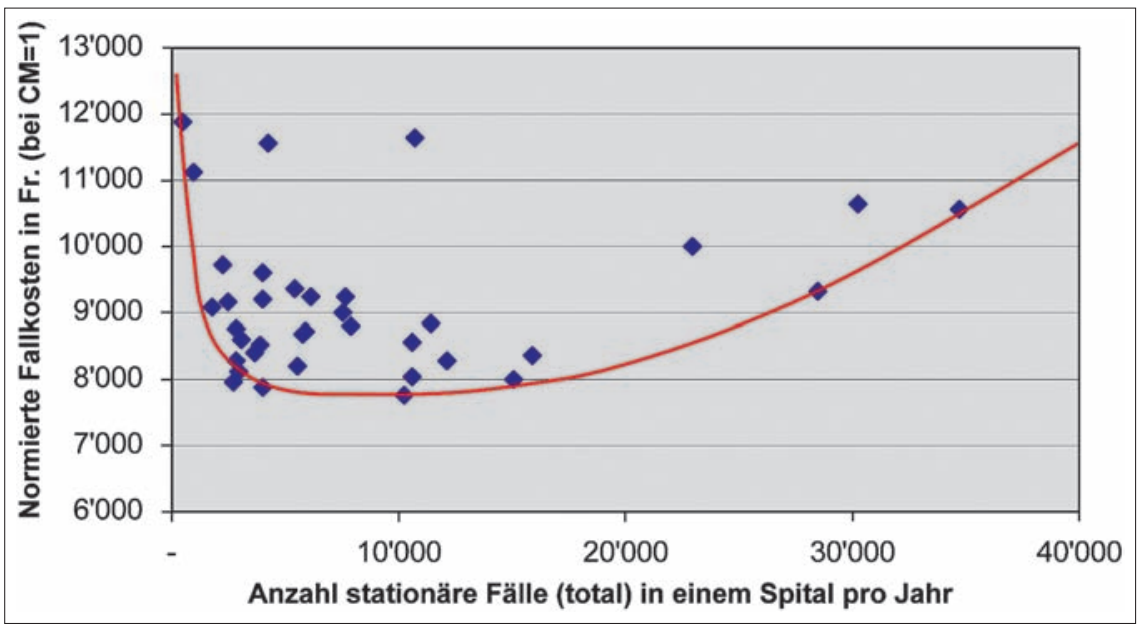

\section{Abbildung 2}

Übersicht über bestehende und zukünftige Mitglieder:

Wachstum von 35 auf $50 \%$ der stationären Fälle.

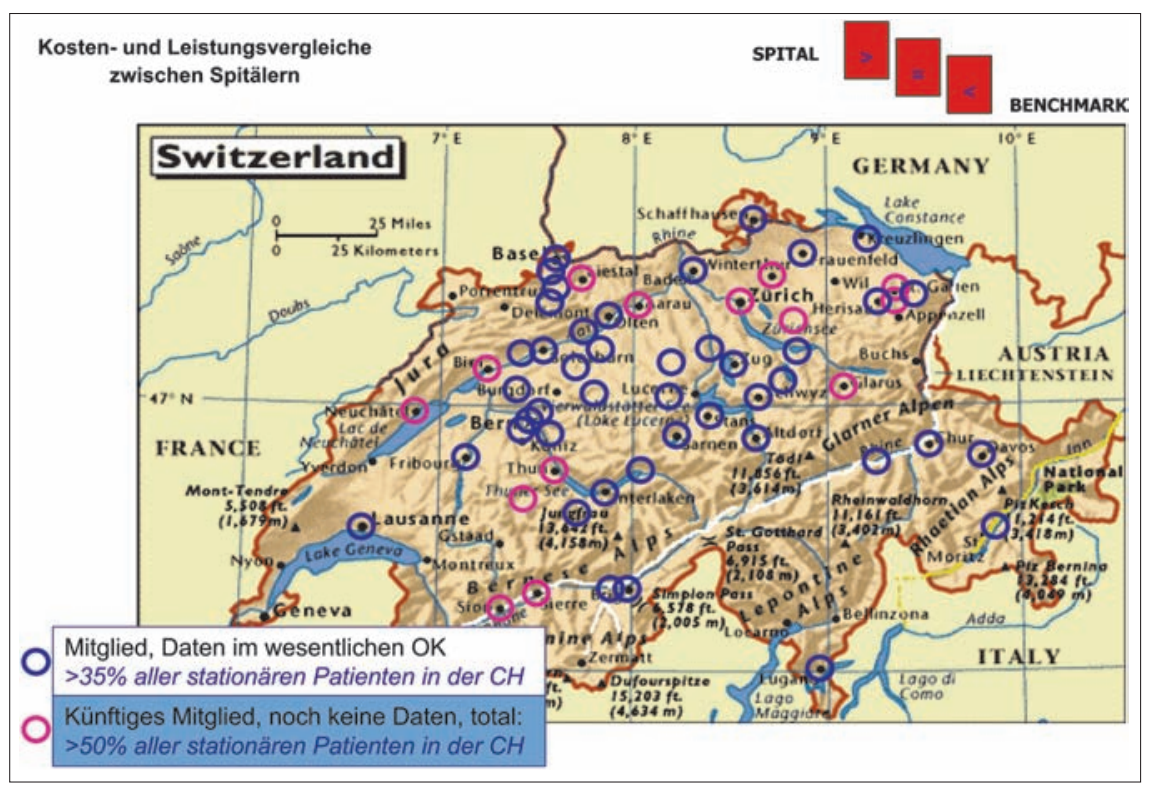

Insgesamt darf festgestellt werden, dass bereits für 2006 ein weitgehend repräsentativer und darum aussagekräftiger Fallkostenbenchmark im Sinne der künftigen KVG-Regelung vorliegt.

\section{Differenzierte Vergleichskategorien}

Die effektiven CMI-normierten 100\%-Fallkosten betragen in den für 2006 erfassten öffentlichen Spitälern im gewichteten Durchschnitt 8690 Franken (ungewichtet 8827 Franken) mit dem Median von 8617 Franken. In den Universitätsspitälern lautet der entsprechende Wert rund 10200 Franken. Es wird sinnvoll sein, für die Universitätsspitäler wegen der zahlreichen
Spezialangebote eine eigene Vergleichskategorie zu schaffen.

Die Streuung aller Fallkosten ist beträchtlich, freilich nicht in dem Masse, wie es die Medien oft darstellen. Ein Teil der Abweichungen lässt sich mit den lokalen Rahmenbedingungen erklären, vorab mit den Lohnunterschieden, auch wenn sie im Gesundheitswesen eher geringer ausfallen als in anderen Branchen. In den Berggebieten wirkt sich die schlechte Verkehrsanbindung an Zentrumsspitäler kostensteigernd aus. Dieser Faktor ist im dichten mittelländischen Spitalnetz kaum relevant. Zusammengefasst darf gefolgert werden, dass die Kostenstreuung dennoch zum grösseren Teil auf echte Effizienzunterschiede in den einzelnen Häusern zurückgeführt werden muss.

Vergleicht man die Spitäler mit den geringsten Fallkosten, so ergibt sich als Funktion der Anzahl Patienten (Grösse) eine Kurve in «U-Form» (Abb. 1), wie sie in ökonomischen Effizienzanalysen oft gefunden wird. Diese Hypothese ist noch weiter zu vertiefen.

Die Stabilität der Resultate, die ersten Ergebnisse der Lernkurve in den beteiligten Unternehmen und allenfalls der Effekt von Privatkliniken werden mit den Daten 2007 zu überprüfen sein.

\section{Verbesserungen als lohnende Herausforderung}

Die vom Verein SpitalBenchmark gewählte Normierung entspricht einem politisch akzeptierten Standardverfahren und dem Strukturziel des neuen KVG. Die Integration von spezialisierten und privaten Spitälern wird gegenwärtig geprüft.

Die Kostenunterschiede zwischen den einzelnen - a priori vom Leistungsspektrum und der Marktstellung her vergleichbaren - Spitälern sind teilweise nachvollziehbar und begründet, mehrheitlich stammen sie aber aus Effizienzunterschieden. Mit einem erweiterten und verfeinerten Tool 2007 sollen Massnahmen zur Verbesserung einfacher voranzutreiben sein (Lernkurve), sofern die entsprechenden Rahmenbedingungen gewährt werden.

Die Erfahrungen in Deutschland haben gezeigt, dass die Einführung eines DRG-Systems mit effektiven Massnahmen zur Qualitätsmessung und Transparenz bei allen wesentlichen Leistungserbringern gekoppelt werden müssen. Erst eine Kombination von aussagekräftigen und objektiven Benchmarks in den Bereichen Kosten und Qualität bringt die Ziele eines neuen DRGTarifsystems zum Tragen. Viele Spitäler der Schweiz sind bereit, diese kombinierte Herausforderung anzunehmen, und haben bereits viel Vorarbeit dazu geleistet. 